ARTIGO ORIGINAL / ORIGINAL ARTICLE

\title{
Análise espacial da taxa de detecção de casos suspeitos de síndrome congênita pelo vírus Zika, Maranhão, 2015 a 2018
}

\author{
Spatial Analysis of Notified Zika Virus Congenital Syndrome, Maranhão, \\ 2015 to 2018
}

\begin{abstract}
Paulo Afonso de Oliveira Falcão Neto' (D), Maria dos Remédios Freitas Carvalho Branco" (D), Silmery da Silva Brito Costa" (D), Ana Patrícia Barros Câmara" (iD, Thayná Millena Nunes França Marques"II (D), Adriana Soraya Araujo" (D), Flávia Helen Furtado Loureiro" (D), José de Jesus Dias Júnior" (D), Maria do Socorro da Silvav (D), Rejane Christine de Sousa Queiroz" (D), Marizélia Rodrigues Costa Ribeiro" (D), Manisha Ann Kulkarni"v" (D), Antônio Augusto Moura da Silva" (D), Alcione Miranda dos Santos" iD
\end{abstract}

RESUMO: Objetivo: Identificar padrões espaciais em casos de lactentes com alterações de crescimento e desenvolvimento relacionadas à infecção pelo vírus Zika e outras etiologias infecciosas (neste trabalho denominado de síndrome congênita pelo vírus Zika), notificados no Maranhão de 2015 a 2018 e sua relação com variáveis socioeconômicas e demográficas. Métodos: Estudo ecológico de casos suspeitos notificados de síndrome congênita pelo vírus Zika nos 217 municípios do Maranhão, Brasil. Calculou-se a autocorrelação espacial pelos índices de Moran local e global (I) univariado e bivariado da taxa de detecção de casos suspeitos de síndrome congênita pelo vírus Zika com índice de desenvolvimento humano municipal, densidade demográfica, índice de Gini e tempo de emancipação político-administrativa dos municípios. O índice de Moran local foi calculado para localizar clusters com autocorrelação espacial significativa. Resultados: Houve autocorrelação espacial na análise univariada da taxa municipal de detecção de casos suspeitos de síndrome congênita pelo vírus Zika ( $\mathrm{I}=0,494 ; \mathrm{p}=0,001)$ e, na análise bivariada, correlação positiva da taxa de detecção de casos suspeitos com índice de desenvolvimento humano municipal $(\mathrm{I}=0,252 ; \mathrm{p}=0,001)$, densidade demográfica $(\mathrm{I}=0,338 ; \mathrm{p}=0,001)$ e tempo de emancipação dos municípios ( $I=0,134 ; p=0,001$ ). Não houve correlação significativa da taxa de detecção de casos suspeitos com o índice de $\operatorname{Gini}(\mathrm{I}=-0,033 ; \mathrm{p}=0,131)$. Cinco clusters de alta detecção de casos suspeitos foram encontrados em áreas distintas do estado. Conclusões: Os municípios com maior índice de desenvolvimento humano municipal, maior densidade demográfica e mais tempo de emancipação políticoadministrativa tiveram mais casos suspeitos notificados de síndrome congênita pelo vírus Zika.

Palavras-chave: Microcefalia. Zika vírus. Análise espacial. Vigilância em saúde pública. Fatores socioeconômicos.

Universidade Federal do Maranhão, Faculdade de Medicina - São Luís (MA), Brasil.

"Universidade Federal do Maranhão, Programa de Pós-Graduação em Saúde Coletiva - São Luís (MA), Brasil.

"'Universidade Ceuma, Curso de Biomedicina - São Luís (MA), Brasil.

vUniversidade Federal do Maranhão, Programa de Pós-Graduação em Saúde e Ambiente - São Luís (MA), Brasil.

vSecretaria Municipal de Saúde - São Luís (MA), Brasil.

vUniversity of Ottawa, School of Epidemiology and Public Health - Ottawa, Canada.

Autor correspondente: Paulo Afonso de Oliveira Falcão Neto. Avenida Deputado Luís Eduardo Magalhães, 350, Condomínio Jardim de Veneto - Torre Veneza, ap. 602, Altos do Calhau, CEP: 65071415, São Luís (MA), Brasil. E-mail: paulofalcao94@hotmail.com Conflito de interesses: nada a declarar - Fonte de financiamento: Este é um subprojeto do projeto "Síndrome congênita pelo Zika vírus, soroprevalência e análise espacial e temporal de vírus Zika e Chikungunya no Maranhão", financiado pela Chamada do Ministério da Ciência, Tecnologia e Inovações (MCTIC)/ Fundo Nacional de Desenvolvimento Científico e Tecnológico (FNDCT), Conselho Nacional de Desenvolvimento Científico e Tecnológico (CNPq)/ Ministério da Educação-Coordenação de Aperfeiçoamento de Pessoal de Nível Superior (MEC-CAPES)/ MS-Decit no 14/2016 — Prevenção e Combate ao vírus Zika. 
ABSTRACT: Objective: To identify spatial patterns in cases of changes in growth and development related to Zika virus infection and other infectious etiologies (denominated Zika virus congenital syndrome in this study) reported in Maranhão from 2015 to 2018 and their relation with socioeconomic and demographic variables. Methods: Ecological study of notified Zika virus congenital syndrome cases in the 217 cities of Maranhão, Brasil. Spatial autocorrelation was calculated using GeoDa 1.14 software and the local and global (I) Moran's index in univariate and bivariate analyses on Zika virus congenital syndrome incidence rate with Municipal Human Development Index (MHDI), population density, Gini coefficient and the cities' time of administrative political emancipation. Local Moran's Index was calculated to identify clusters with significant spatial autocorrelation. Results: Spatial autocorrelation was checked in univariate analysis of the incidence rate of Zika virus congenital syndrome $(I=0,494 ; p=0,001)$ and positive correlation in bivariate analysis of the incidence rate with Municipal Human Development Index ( $I=0,252 ; p=0,001)$, population density $(I=0,338 ; p=0,001)$ and the cities' time of administrative political emancipation $(\mathrm{I}=0,134 ; \mathrm{p}=0,001)$. The correlation between incidence rate with Gini coefficient was not significant $(I=-0,033 ; p=0,131)$. Five high-incidence clusters were found in distinct areas of the state. Conclusions: Cities with higher MHDI, higher population density and more years of administrative political emancipation had more cases of Zika virus congenital syndrome notified.

Keywords: Microcephaly. Zika Virus. Spatial analysis. Public health surveillance. Socioeconomic factors.

\section{INTRODUÇÃO}

O vírus Zika (ZKV) é transmitido pela picada da fêmea infectada do Aedes aegypti, por via sexual e transplacentária. No Brasil, o ZKV foi introduzido na região Nordeste do país em 2014, desencadeando um surto em 2015, acompanhado do surgimento de casos de síndrome congênita pelo Zika vírus $(\mathrm{SCZV})^{1}$. O termo SCZV refere-se às manifestações congênitas causadas pela exposição intrauterina ao $Z \mathrm{KV}$, que incluem microcefalia, convulsões, contraturas musculares, anomalias auditivas e oculares². No período de 2015 a 2018, o Brasil notificou 17.041 casos de alterações no crescimento e no desenvolvimento relacionadas à infecção pelo ZKV e outras etiologias infecciosas ${ }^{3}$.

A região Nordeste do Brasil tem os menores índices de desenvolvimento humano municipal (IDHM) e maior desigualdade de renda (altos índices de Gini), possuindo ao mesmo tempo a maior detecção de casos suspeitos de $\mathrm{SCZV}^{1}$. O Maranhão, localizado nessa região do país, é o penúltimo no ranking de IDH entre os estados brasileiros ${ }^{4}$ e o sétimo estado da região com o maior número de notificações de alterações de crescimento e desenvolvimento relacionadas à infecção pelo ZKV e outras etiologias infecciosas até $2018^{3}$.

As técnicas de análise espacial permitem averiguar a distribuição espacial de agravos, doenças e situações de risco, o que possibilita detectar áreas vulneráveis, conhecer mais detalhadamente os padrões das condições de saúde de uma população, bem como evidenciar disparidades ${ }^{5}$. Com o avanço de métodos estatísticos e técnicas de análise espacial, estudos sobre a distribuição geográfica de anomalias congênitas e seus fatores etiológicos vêm demonstrando sua importância para a saúde pública no Brasil e no mundo 6 . 
Neste estudo, considerando-se que a SCZV teve maior ocorrência em áreas mais pobres do país, levantou-se a hipótese de que no Maranhão tenha havido mais registros de casos de SCZV em municípios com menores IDHM e maiores índices de Gini; naqueles com maior densidade demográfica em razão da possibilidade de terem maiores índices de infestação vetorial; assim como nos municípios com mais tempo de emancipação político-administrativa, por possuírem melhor estrutura de vigilância epidemiológica. O objetivo deste estudo foi identificar padrões espaciais de casos suspeitos de SCZV notificados no Maranhão de 2015 a 2018 e sua relação com as variáveis socioeconômicas e demográficas já citadas.

\section{MÉTODOS}

Estudo ecológico com análise espacial de casos suspeitos notificados de alterações de crescimento e desenvolvimento relacionadas à infecção pelo ZKV e outras etiologias infecciosas (neste trabalho denominado de SCZV) nos municípios do Maranhão, de janeiro de 2015 a novembro de 2018.

A área do estudo compreendeu o estado do Maranhão, que possui 217 municípios e localiza-se na região Nordeste do Brasil. Em 2018 sua densidade demográfica era de 22,54 habitantes $/ \mathrm{km}^{2}$ e sua população era de 7.035 .055 habitantes ${ }^{3}$.

Os casos suspeitos de SCZV foram notificados, ao nascimento ou posteriormente, em um sistema específico denominado Registro de Eventos em Saúde Pública (RESP). Realizou-se a coleta de dados do RESP no período de abril a novembro de 2018. O RESP é um sistema de notificação online desenvolvido pelo Ministério da Saúde com a finalidade de registrar as emergências em saúde pública. Para a notificação imediata de casos suspeitos de microcefalias foi construído pelo Departamento de Informática do Sistema Único de Saúde (DATASUS) um formulário online no RESP, denominado de RESP-Microcefalias e utilizado para notificar casos suspeitos de microcefalia e/ ou alterações do sistema nervoso central (SNC) possivelmente associadas a infecções congênitas.

Neste estudo, o desfecho de interesse foi a taxa de detecção de casos suspeitos de SCZV por município, calculada com base na razão entre o número de casos suspeitos notificados e o número de nascidos vivos no período de janeiro de 2015 a novembro de 2018 multiplicada por 10 mil habitantes. Para o estado do Maranhão foi calculada a taxa mensal de detecção de casos suspeitos. O número de nascidos vivos para cada município foi obtido do banco de dados do Sistema de Informação sobre Nascidos Vivos (SINASC).

As variáveis demográficas e socioeconômicas utilizadas foram: data e município de nascimento, obtidos no RESP; IDHM e índice de Gini, extraídos do censo de 2010; densidade demográfica, com a estimativa populacional de 2018, e ano de emancipação político-administrativa dos municípios retirados do site do Instituto Brasileiro de Geografia e Estatística (IBGE) ${ }^{4}$. Para o cálculo do tempo de emancipação, utilizou-se como referência o ano de 2015.

O IDHM consiste em três indicadores: renda, longevidade e educação, que variam de zero a um. A densidade demográfica do município é definida pelo número de habitantes 
por quilômetro quadrado. O índice de Gini mede a desigualdade social com base na renda per capita; varia de zero a um e, quanto mais próximo de um, maior a desigualdade social ${ }^{4}$.

Os casos notificados de SCZV foram agregados por município, utilizando-se a malha municipal digital do estado do Maranhão. Todos os municípios foram incluídos na análise espacial, independentemente da notificação de casos. A taxa de detecção de casos suspeitos foi suavizada pelo método de estimativa bayesiana local.

Para verificar se municípios vizinhos exibiam tendências semelhantes na taxa de detecção de casos suspeitos de SCZV, o índice de Moran global (I) univariado foi calculado como uma medida de autocorrelação espacial. Para avaliar a dependência espacial entre a taxa de detecção de casos suspeitos de SCZV e as variáveis densidade demográfica, IDHM, índice de Gini e tempo de emancipação político-administrativa, o índice de Moran global bivariado foi calculado. O índice de Moran global caracteriza-se por fornecer uma medida geral da associação espacial existente no conjunto de dados ${ }^{7}$. Varia de $-1 \mathrm{a}+1$, e os valores próximos de zero indicam inexistência de autocorrelação espacial; valores positivos indicam autocorrelação espacial positiva; e valores negativos indicam autocorrelação espacial negativa. Para aqueles que apresentam padrão espacial, os valores são positivos, próximos a 1, ou seja, áreas vizinhas apresentam comportamento similar. Já valores próximos a -1 indicam autocorrelação espacial negativa, isto é, o valor do atributo em regiões vizinhas é contrastante entre si ${ }^{8}$.

Para identificar clusters de municípios com autocorrelação e correlação espacial significativa da taxa de detecção de casos suspeitos de SCZV, o índice de Moran local foi calculado e demonstrado no Lisa Cluster Map. O índice de Moran local produz um valor específico para cada área, permitindo a identificação de aglomerados de áreas com padrões significativos de associação espacial. Esse índice classificou os municípios em função do nível de significância dos valores de seus índices locais em: alta/alta (município com valor alto, circundado por vizinhos com valor alto) e baixa/baixa, indicando pontos de associação espacial positiva ou similar aos vizinhos; e alta/baixa e baixa/alta, indicando pontos de associação espacial negativa, ou seja, a localização possui vizinhos com valores distintos ${ }^{8}$.

Para a análise espacial foi utilizado o software GeoDa versão 1.14, e o nível de significância considerado para a autocorrelação espacial foi $\mathrm{p}<0,05$.

O projeto foi aprovado pelo Comitê de Ética em Pesquisa do Hospital Universitário da Universidade Federal do Maranhão (HUUFMA), sob parecer n 2.111.125.

\section{RESULTADOS}

No RESP foram registrados 426 casos de SCZV durante o período do estudo. Desses, quatro foram duplicatas, de modo que se analisaram 422. No estado, a taxa de detecção de casos suspeitos de SCZV por 10 mil nascidos vivos foi de 15,99 em 2015; de 13,84 em 2016; de 5,75 em 2017 e de 1,49 em 2018. Houve notificação de casos suspeitos de SCZV em 111 municípios, sendo a maior taxa de detecção de casos suspeitos em Alcântara — 62,97 / 10.000 nascidos vivos (dados não apresentados em tabelas). As crianças começaram a nascer em 
janeiro de 2015, embora o surto de SCZV só tenha sido percebido em outubro do mesmo ano. O pico de casos suspeitos notificados ocorreu em dezembro de 2015 e houve queda após o primeiro trimestre de 2016, mais acentuada após fevereiro de 2018 (Figura 1).

O índice de Moran global univariado mostrou autocorrelação espacial positiva significativa para a taxa de detecção de casos suspeitos estimada de 2015 a 2018. O índice de Moran global bivariado mostrou correlação positiva da taxa de detecção de casos suspeitos de SCZV de 2015 a 2018 com densidade demográfica, IDHM e tempo de emancipação dos municípios. Não foi verificada correlação espacial estatisticamente significante com o índice de GINI (Tabela 1).

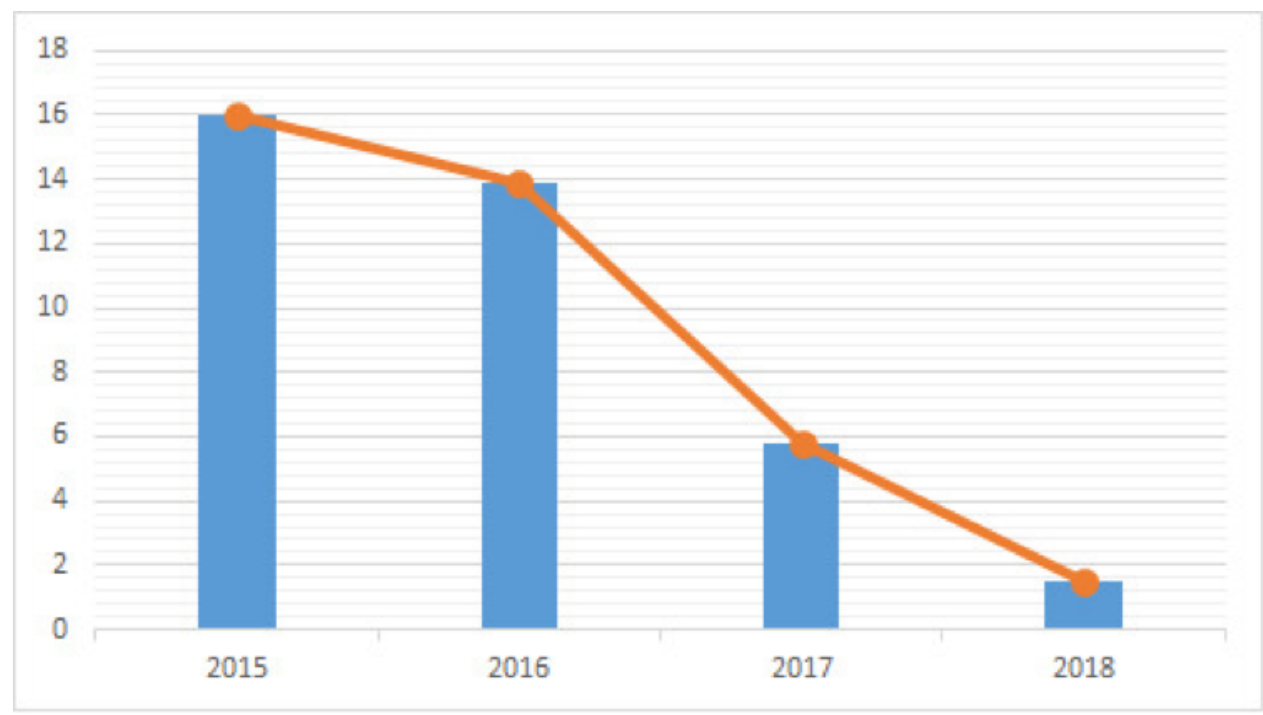

Figura 1. Taxa anual de detecção de casos suspeitos de síndrome congênita pelo Zika vírus por 10 mil nascidos vivos no Maranhão, de 2015 a 2018.

Tabela 1. Análise univariada da taxa municipal de detecção de casos suspeitos de síndrome congênita pelo Zika vírus de 2015 a 2018 e análise bivariada da taxa de detecção de casos suspeitos com tempo de emancipação do município, densidade demográfica, índice de Gini e índice de desenvolvimento humano municipal nos 217 municípios do Maranhão.

\begin{tabular}{|l|c|c|}
\hline \multicolumn{3}{|c|}{ Análise univariada } \\
\hline \multicolumn{1}{|c|}{ Andice de Moran Global } & p-valor \\
\hline \multicolumn{2}{|c|}{ Análise bivariada com a taxa de detecção de casos suspeitos entre 2015 e 2018 } \\
\hline \multicolumn{2}{|c|}{0,494} & ín-valor \\
\hline Tempo de emancipação do município & Índice de Moran Global & 0,001 \\
\hline Densidade demográfica & 0,134 & 0,001 \\
\hline Índice de Gini & 0,338 & 0,131 \\
\hline Índice de desenvolvimento humano municipal & $-0,033$ & 0,001 \\
\hline
\end{tabular}


O índice de Moran local identificou clusters formados por 18 municípios com alta taxa de detecção de casos suspeitos de SCZV no norte (onde se localiza a capital do estado), oeste, sudoeste e sul; clusters formados por 12 municípios com altas taxas de detecção de casos suspeitos de SCZV e com maior tempo de emancipação político-administrativa, assim como clusters (nove municípios) com alta densidade demográfica foram identificados ao norte; e clusters (21 municípios) com alta taxa de detecção de casos suspeitos de SCZV e altos valores de IDHM foram verificados no norte, oeste e sudoeste do estado (Figura 2) (Tabela 2).

\section{DISCUSSÃO}

Identificou-se um padrão espacial significativo da distribuição dos casos suspeitos notificados de SCZV no estado, com clusters de alta detecção de casos suspeitos especialmente no entorno da capital do estado. Padrão semelhante foi verificado no Maranhão por Costa et al. ${ }^{9}$ em relação à taxa de incidência de Zika. Os clusters de Zika podem se tornar clusters da SCZV aproximadamente três meses após o surto em uma determinada região ${ }^{10}$.

Em estudo realizado no estado nordestino do Rio Grande do Norte, Cunha et al. ${ }^{11}$ encontraram maior notificação de casos de ZKV em municípios com maior renda média, provavelmente com melhor infraestrutura e maior acesso aos serviços de saúde, como a
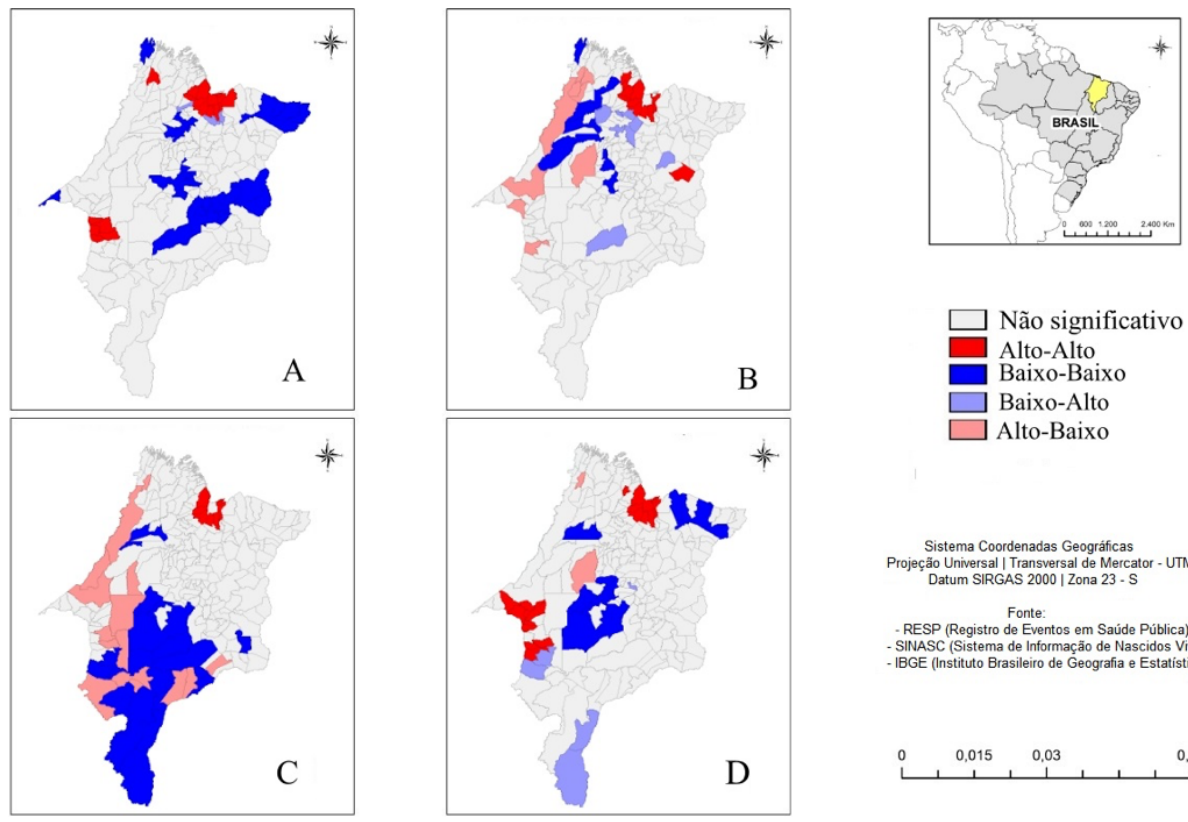

Sistema Coordenadas Geográicas

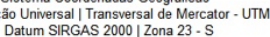

Fonte

- RESP (Registro de Eventos em Saúde Pública): IBGE (Instituto Brasileiro de Gạo de Nascidos Vivos:

(1)

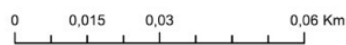

Figura 2. Lisa Cluster Map da taxa de detecção de casos suspeitos de síndrome congênita pelo Zika vírus $(A)$ e das correlações espaciais entre taxa de detecção de casos suspeitos de síndrome congênita pelo Zika vírus e tempo de emancipação dos municípios (B), densidade demográfica (C) e índice de desenvolvimento humano municipal (D). Maranhão, 2015-2018. 
capital Natal. Tal discrepância foi atribuída à maior capacidade de reconhecimento e notificação dos casos.

A correlação espacial positiva entre as taxas municipais de detecção de casos suspeitos de SCZV e IDHM é explicada provavelmente pela melhor estrutura para registro de casos nos municípios com melhores IDH. Entretanto, dois estudos realizados em Recife/PE verificaram maior risco de SCZV em distritos com IDH baixo ${ }^{12}$ e em locais com baixas condições de moradia $^{13}$. Uma análise da relação entre fatores socioeconômicos e casos suspeitos de SCZV no Brasil não verificou associação da taxa de detecção de casos suspeitos com IDHM, mas atestou forte correlação da distribuição dos casos com maior índice de pobreza. O mesmo estudo infere que fatores socioeconômicos tiveram influência no desenvolvimento no surto de SCZV que se iniciou no Nordeste ${ }^{14}$.

Tabela 2. Características dos clusters da taxa municipal de detecção de casos suspeitos de síndrome congênita pelo Zika vírus. Maranhão, Brasil, 2015-2018.

\begin{tabular}{l|l} 
Clusters & Quantidade de municípios nos clusters \\
\hline
\end{tabular}

Taxa de detecção de casos suspeitos

\begin{tabular}{|l|c|}
\hline Alto-Alto & 18 \\
\hline Baixo-Baixo & 39 \\
\hline Baixo-Alto & 3 \\
\hline Alto-Baixo & 0 \\
\hline Taxa de deteção de casos suspeitos x Tempo de emancipação político-administrativa
\end{tabular}

Taxa de detecção de casos suspeitos x Tempo de emancipação político-administrativa

\begin{tabular}{l|c}
\hline Alto-Alto & 12 \\
\hline Baixo-Baixo & 7 \\
\hline Baixo-Alto & 8 \\
\hline Alto-Baixo & 9 \\
\hline
\end{tabular}

Taxa de detecção de casos suspeitos $x$ Densidade demográfica

\begin{tabular}{l|c}
\hline Alto-Alto & 9 \\
\hline Baixo-Baixo & 21 \\
\hline Baixo-Alto & 0 \\
\hline Alto-Baixo & 16 \\
\hline
\end{tabular}

Taxa de detecção de casos suspeitos $x$ IDHM

\begin{tabular}{l|c}
\hline Alto-Alto & 21 \\
\hline Baixo-Baixo & 15 \\
\hline Baixo-Alto & 5 \\
\hline Alto-Baixo & 2 \\
\hline
\end{tabular}

IDHM: índice de desenvolvimento humano municipal. 
Encontrou-se correlação positiva da taxa de detecção de casos suspeitos de SCZV com a densidade demográfica. Amaral et al..$^{15}$ encontraram maior taxa de incidência de microcefalia em municípios brasileiros com maior população, índice de mobilidade e cobertura da atenção básica, o que pode ser explicado pela maior facilidade de transmissão de infecções congênitas e pela maior facilidade de diagnóstico (no caso da cobertura da atenção básica).

Corroborando nossos achados, uma análise da distribuição espacial de anomalias congênitas no estado do Paraná verificou associação significativa com o grau de urbanização, ressaltando a maior capacidade de vigilância em saúde em áreas urbanizadas ${ }^{16}$, enquanto Mocelin et al. encontraram menor número de notificações de SCZV em municípios rurais do Espírito Santo ${ }^{17}$.

Não foi verificada correlação espacial estatisticamente significativa da taxa de detecção de casos suspeitos com o índice de Gini. No Brasil, Ali et al. ${ }^{18}$ demonstraram uma forte correlação entre SCZV e menor produto interno bruto (PIB) per capita nas unidades federativas e postularam que a proximidade de áreas socioeconomicamente distintas pode facilitar a transmissão de ZKV, ultrapassando barreiras sociais. Nas Américas, Gardner et al. ${ }^{19}$ também verificaram correlação positiva com a densidade populacional, associação negativa entre PIB per capita e transmissão de ZKV.

$\mathrm{O}$ achado de mais registros de SCZV em municípios com maior IDHM, maior densidade demográfica e mais tempo de emancipação política sugere que municípios com essas características possuam melhor estrutura de serviços de saúde, incluindo a vigilância em saúde. Enquanto o simples fato de emancipar um município não garante seu desenvolvimento $^{20}$, supõe-se que embora a SCZV seja uma doença da pobreza os municípios com menor IDH, menor densidade demográfica e menos tempo de emancipação não tenham conseguido estruturar a vigilância epidemiológica e assim garantir a notificação dos casos.

Outro aspecto a ser considerado é o fato de que os ambientes mais populosos, caracterizados por crescimento urbano desordenado, condições precárias de moradia e serviço de saneamento básico ineficaz como a eliminação inadequada de resíduos sólidos podem favorecer os riscos de infecções transmitidas por vetores que se multiplicam nessas áreas vulneráveis e contribuem para o aumento de $\operatorname{casos}^{21}$.

Entre as limitações do estudo, destaca-se que não foi realizada análise espacial multivariada para descartar a colinearidade entre densidade populacional e IDHM. Foram incluídas todas as notificações, o que representa uma limitação quanto à especificidade do desfecho. Outra limitação é a utilização de dados (Gini, IDHM) do censo de 2010 dada a indisponibilidade de indicadores socioeconômicos mais recentes. Ressalta-se que a utilização da unidade de análise espacial não foi pontual pelo endereço de residência do caso suspeito, ou seja, houve certo grau de imprecisão na análise. Adicionalmente, a unidade de análise espacial em nível municipal não permite identificar as disparidades intramunicipais. Sabe-se que a baixa frequência dos casos contribui para estimativas imprecisas e que a baixa magnitude da correlação espacial encontrada é uma evidência a favor dessa interpretação. Além disso, entre as limitações próprias dos estudos ecológicos, existe a possibilidade de falácia ecológica. 
Este estudo chama a atenção para a necessidade de se melhorar a qualidade da vigilância em saúde nos municípios do estado. Ressaltam-se como pontos fortes a contribuição para a compreensão dos determinantes relacionados aos casos de SCZV e a identificação de áreas prioritárias por meio da análise espacial, que considera as desigualdades em espaços geográficos.

\section{AGRADECIMENTOS}

Ao CNPq o financiamento do projeto "Síndrome congênita pelo Zika vírus, soroprevalência e análise espacial e temporal de vírus Zika e Chikungunya no Maranhão”.

\section{REFERÊNCIAS}

1. Barbeito-Andrés J, Schuler-Faccini L, Garcez PP. Why is congenital Zika syndrome asymmetrically distributed among human populations? PLoS Biol 2018; 16 (8): e2006592. https:// doi.org/10.1371/journal.pbio.2006592

2. Moore CA, Staples JE, Dobyns WB, Pessoa A, Ventura $\mathrm{CV}$, Fonseca $\mathrm{EB}$, et al. Characterizing the pattern of anomalies in congenital Zika syndrome for pediatric clinicians. JAMA Pediatr 2017; 171 (3): 288-95. https: / / doi.org/10.1001/jamapediatrics.2016.3982

3. Ministério da Saúde. Secretaria de Vigilância em Saúde. Monitoramento integrado de alterações no crescimento e desenvolvimento relacionados à infecção pelo vírus Zika e outras etiologias infecciosas, até a Semana Epidemiológica 52 de 2018. Ministério da Saúde [Internet]. 2019 [acessado em 22 dez. 2019]. Disponível em: http: / portalarquivos2.saude.gov.br/ images/pdf/2019/marco/22/2019-001.pdf

4. Instituto Brasileiro de Geografia e Estatística. Conheça cidades e estados do Brasil [Internet]. 2019 [acessado em 10 nov. 2019]. Disponível em: https:// cidades. ibge.gov.br/

5. Almeida Filho N, Barreto ML. Epidemiologia \& Saúde: fundamentos, métodos, aplicações. Rio de Janeiro: Guanabara Koogan; 2012.

6. Lima LMM, Melo ACO, Vianna RPT, Moraes RM. Análise espacial das anomalias congênitas do sistema nervoso. Cad Saúde Colet 2019; 27 (3): 257-63. https: / doi.org/10.1590/1414-462X201900030313

7. Druck S, Carvalho MS, Câmara G, Monteiro AMV. Spatial analysis of geographic data [Internet]. Brasília: Embrapa Cerrados; 2004. [acessado em 7 jan. 2021]. Disponível em: http: / /ivimagens.sct.embrapa.br/ amostras/00075490.pdf
8. Câmara G, Carvalho MS, Cruz OG, Correa V. Análise espacial de áreas. In: Druck, S, Carvalho MS, Câmara G, Monteiro AVM, editores. Análise espacial de dados geográficos. Brasília: São Paulo: Instituto Nacional de Pesquisas Espaciais; 2002.

9. Costa SDSB, Branco MDRFC, Aquino Junior J, Rodrigues ZMR, Queiroz RCS, Araujo AS. Spatial analysis of probable cases of dengue fever, chikungunya fever and zika virus infections in Maranhao State, Brazil. Rev Inst Med Trop Sao Paulo 2018; 60: e62. https: / / doi.org/10.1590/S1678-9946201860062

10. Vissoci JRN, Rocha TAH, Silva NCD, de Sousa Queiroz RC, Thomaz EBAF, Amaral PVM, et al. Zika virus infection and microcephaly: Evidence regarding geospatial associations. PLoS Negl Trop Dis 2018; 12 (4): e0006392. https:// doi.org/10.1371/journal. pntd.0006392

11. Cunha LS, Medeiros WR, Lima Junior FAV, Pereira SA. Relationship between social inequality indicators and the spatial distribution of Zika Virus cases. Cien Saude Colet 2020; 25 (5): 1839-50. https: / / doi. org/10.1590/1413-81232020255.34642019

12. Souza AI, Siqueira MT, Ferreira ALCG, Freitas CU, Bezerra ACV, Ribeiro AG, et al. Geography of microcephaly in the Zika Era: a study of newborn distribution and socio-environmental indicators in Recife, Brazil, 2015-2016. Public Health Rep 2018; 133 (4): 461-71. https:/ / doi.org/10.1177/0033354918777256

13. Souza WV, Albuquerque MFPM, Vazquez E, Bezerra LCA, Mendes ADCG, Lyra TM, et al. Microcephaly epidemic related to the $Z$ ika virus and living conditions in Recife, Northeast Brazil. BMC Public Health 2018; 18 (1): 130. https: / / doi.org/10.1186/s12889-018-5039-Z 
14. Campos MC, Dombrowski JG, Phelan J, Marinho CRF, Hibberd M, Clark TG, et al. Zika might not be acting alone: using an ecological study approach to investigate potential co-acting risk factors for an unusual pattern of microcephaly in Brazil. PLoS One 2018; 13 (8): e0201452. https: / / doi.org/10.1371/journal. pone. 0201452

15. Amaral P, Carvalho LR, Rocha TAH, Silva NC, Vissoci JRN. Geospatial modeling of microcephaly and zika virus spread patterns in Brazil. PLoS One 2019; 14 (9): e0222668. https://doi.org/10.1371/journal. pone. 0222668

16. Freire MHS, Barros APM, Andrade L, Nihei OK, Fontes KB. Análise geoespacial dos nascimentos com anomalias congênitas, Paraná, 2008-2015: estudo ecológico. Rev Bras Enferm 2020; 73 (3): e20180741. https: / / doi.org/10.1590/0034-7167-2018-0741

17. Mocelin HJS, Catão RC, Freitas PSS, Prado TN, Bertolde AI, Castro MC, et al. Analysis of the spatial distribution of cases of Zika virus infection and congenital Zika virus syndrome in a state in the southeastern region of Brazil: Sociodemographic factors and implications for public health. Int J Gynaecol Obstet 2020; 148 (Suppl 2): 61-9. https: / / doi.org/10.1002/ijgo.13049

18. Ali S, Gugliemini O, Harber S, Harrison A, Houle L, Ivory J, et al. Environmental and social change drive the explosive emergence of Zika virus in the Americas. PLoS Negl Trop Dis 2017; 11 (2): e0005135. https: / / doi.org/10.1371/journal.pntd.0005135

19. Gardner LM, Bóta A, Gangavarapu K, Kraemer MUG, Grubaugh ND. Inferring the risk factors behind the geographical spread and transmission of Zika in the Americas. PLoS Negl Trop Dis 2018; 12 (1): e0006194. https: / / doi.org/10.1371/journal.pntd.0006194
20. Magalhães J. Emancipação político-administrativa dos municípios no Brasil. In: Carvalho A, Albuquerque C, Mota J, Piancastelli M. Dinâmica dos municípios [Internet]. Brasília:IPEA; 2007 [acessado em $22 \mathrm{dez}$. 2019]. Disponível em: http: / / www.ipea.gov.br/portal/ images/stories/PDFs/livros/Capitulo1_30.pdf

21. Almeida LS, CotaALS, RodriguesDF. Sanitation, Arboviruses, and Environmental Determinants of Disease: impacts on urban health. Cien Saude Colet 2020; 25 (10): 3857-68. https: / / doi.org/10.1590/1413-812320202510.30712018

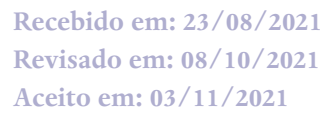

Contribuição dos autores: Falcão Neto, P.A.O.: Conceituação, Análise Formal, Escrita - Revisão e Edição. Branco, M.R.F.C.: Conceituação, Análise Formal, Administração do Projeto, Escrita - Primeira Redação. Costa, S.S.B.: Conceituação, Análise Formal, Escrita - Primeira Redação. Câmara, A.P.B.: Conceituação, Análise Formal, Escrita - Primeira Redação. Marques, T.M.N.F.: Conceituação, Curadoria de Dados, Escrita - Primeira Redação. Araujo, A.S.: Conceituação, Escrita - Primeira Redação. Loureiro, F.H.F: Conceituação, Escrita - Primeira Redação. Dias Júnior, J.J.: Conceituação, Escrita - Primeira Redação. Silva, M.S.: Conceituação, Escrita - Primeira Redação. Queiroz, R.C.S.: Conceituação, Escrita - Primeira Redação. Ribeiro, M.R.C.: Escrita - Revisão e Edição. Kulkarni, M.A.: Escrita - Revisão e Edição. Silva, A.A.M.: Obtenção de Financiamento, Administração do Projeto, Escrita - Revisão e Edição. Santos, A.M.: Conceituação, Administração do Projeto, Escrita - Revisão e Edição. 